\title{
Synthesis of thiolated and acrylated nanoparticles using thiol-ene click chemistry: towards novel mucoadhesive materials for drug delivery ${ }^{\dagger}$
}

Cite this: RSC Advances, 2013, 3 12275

Received 28th April 2013,

Accepted 21st May 2013

DOI: $10.1039 / \mathrm{c} 3 \mathrm{ra} 42093 \mathrm{k}$

www.rsc.org/advances

\author{
Anna Štorha, ${ }^{a}$ Ellina A. Mun ${ }^{b}$ and Vitaliy V. Khutoryanskiy*b
}

Thiol- and acrylate-functionalized nanoparticles have been synthesized from pentaerythritol tetrakis(3mercapto-propionate) and pentaerythritol tetraacrylate using thiol-ene click chemistry. Using Raman and ${ }^{1} \mathrm{H}$ NMR spectroscopy as well as Ellman's assay, it was demonstrated that excess pentaerythritol tetraacrylate in the feed mixture led to nanoparticles with free acrylate groups on their surface, whereas nanoparticles with thiolated surfaces could be synthesized using feed mixtures with excess pentaerythritol tetrakis(3-mercapto-propionate). The possibility of fluorescent labelling of thiolated nanoparticles has been demonstrated through their reaction with fluorescein-5-maleimide. The thiolated nanoparticles were found to be mucoadhesive and exhibited retention on mucosal surface of porcine urinary bladder.

\section{Introduction}

Hydro-thiolation of $\mathrm{C}=\mathrm{C}$ bonds, known as thiol-ene "click" reactions, has recently become a very popular approach to synthesize various polymeric materials. These reactions are highly versatile and can be achieved using radical conditions (often involving photochemical initiation); catalytic processes mediated by nucleophiles, acids and bases or can simply be solvent promoted in the presence of highly polar media such as $N, N$-dimethylformamide (DMF). ${ }^{1,2}$

To achieve complete conversion of thiol-ene click reactions and to maximize the mechanical properties of materials, thioland alkene-based precursors are often used in stoichiometric $(1: 1)$ ratio. However, in these cases the resultant materials typically have non-reactive surfaces. Recently Carlborg et al. ${ }^{3}$ reported the first use of non-stoichiometric thiol-to-ene ratios resulting in materials with residual unreacted functional groups both in the bulk and on the surface. They found a number of advantages for these materials, such as tunable mechanical properties and possibilities for their surface modifications.

The development of novel materials with active functional groups on their surface or in the bulk is of significant interest in a number of biomedical areas, including drug delivery and imaging. In particular, this approach is highly relevant to

\footnotetext{
${ }^{a}$ Rīga Stradinš University, Faculty of Pharmacy, Riga, LV-1007, Latvia

${ }^{b}$ Reading School of Pharmacy, University of Reading, Reading, United Kingdom. E-mail: v.khutoryanskiy@reading.ac.uk; Fax: +44(0)1183784703;

Tel: +44(0)118378669

$\dagger$ Electronic supplementary information (ESI) available: Details of feed mixtures for thiol-ene click reactions, ${ }^{1} \mathrm{H}$ NMR spectra of nanoparticles, fluorescence spectra of labelled nanoparticles, results on hydrolytic degradation. See DOI: $10.1039 / \mathrm{c} 3 \mathrm{ra} 42093 \mathrm{k}$
}

nanomaterials that could potentially be post-functionalized (e.g. PEGylation, conjugation with fluorescent dyes or drug molecules). ${ }^{4-6}$

The presence of unreacted thiol groups on the surface of nanoparticles resulting from non-stoichiometric thiol-ene reactions could also provide materials with mucoadhesive properties, i.e. the ability to stick and retain on mucosal surfaces due to disulfide bond formation with cysteine residues of mucins. ${ }^{7-9}$

The majority of studies on the development of mucoadhesive thiol-functionalised nanoparticles for drug delivery have reported the use of polymer complexation involving thiolated water-soluble polymers. This method was reported by Bernkop-Schnürch and co-workers and utilised various forms of complex formation: ionic gelation of thiolated chitosan with tripolyphosphate, ${ }^{10}$ or hydrogen-bonded interpolymer complexes of thiolated poly(acrylic acid) with poly $(N$-vinyl pyrrolidone). ${ }^{11}$

Recently we reported the self-condensation of 3-mercaptopropyltrimetoxysilane in dimethylsulfoxide as a novel method for the synthesis of thiolated silica nanoparticles. ${ }^{12,13}$ These nanoparticles exhibited excellent mucoadhesive potential and retention on ocular surfaces; however, they were non-porous and non-swellable, which limited the possibilities for their drug loading.

The present study reports the first application of thiol-ene click reactions with non-stoichiometric ratio of reagents for the synthesis of thiol- and acrylate-functionalized nanoparticles. Thiolated nanoparticles synthesized were found to be adhesive to mucosal lining of porcine urinary bladder. The particles were also swellable in organic solvents and showed 
some signs of biodegradation in aqueous media, which makes them promising for application in drug delivery.

\section{Experimental section}

\section{Materials}

Pentaerythritol tetrakis(3-mercaptopropionate) (PEMP, purity $\geqslant 95 \%$ ), pentaerythritol tetraacrylate (PETA, containing 350 ppm monomethyl ether hydroquinone as inhibitor), N,Ndimethylformamide (ACS reagent, $\geqslant 99.8 \%$ ), 5,5'-dithiobis(2nitrobenzoic acid) (DTNB $\geqslant 98 \%$ (TLC)), cysteine hydrochloride $(\geqslant 98 \%$ (TLC)), FITC-dextran (20 kDa), fluorescein-5maleimide, dimethyl sulfoxide-d6 (DMSO-d6) and chemicals used for preparation of artificial urine were purchased from Sigma Aldrich, Inc. (UK) and used as received.

\section{Synthesis of nanoparticles}

Different concentrations of PETA and PEMP in DMF were used to synthesize nanoparticles $(1,5,10,20,30,40$ and 50 vol\%). PETA and PEMP in DMF were mixed in different ratios and the reaction was allowed to proceed for $24 \mathrm{~h}$. The reaction mixtures were stirred using a magnetic stirrer until gelation or for $24 \mathrm{~h}$ for the samples without gelation. Depending on the composition of the mixture the formation of either transparent gel or solutions was observed as a result of thiol-ene click reaction. The outcomes of each reaction are summarized in Table 1S (ESI $\dagger$ ). $2 \mathrm{~mL}$ deionized water was added to each mixture that did not result in formation of a gel. This led to the immediate formation of milky suspension of nanoparticles (sols), which were subsequently purified using dialysis through cellulose-based membranes with MWCO 12-14 kDa (Medicell International Ltd, UK). The dialysis tubing with solution was placed in $5 \mathrm{~L}$ beaker filled with deionized water and was stirred continuously for $24 \mathrm{~h}$ ( 6 changes of water in total). The purified nanoparticles were stored in aqueous dispersions in sealed containers at $4{ }^{\circ} \mathrm{C}$. All measurements with dispersions of nanoparticles in water were performed immediately after their purification. Some portions of nanoparticles were also freeze-dried immediately after purification to use dry samples for analysis.

\section{Characterization of nanoparticles}

Dynamic light scattering (DLS) and zeta-potential measurements were performed with dilute dispersions of nanoparticles at $25{ }^{\circ} \mathrm{C}$ using Nano-S Zetasizer (Malvern Instruments, UK). Transmission electron microscopy (TEM) images of nanoparticles were acquired using a Philips CM20 Analytical TEM (acceleration voltage is $200 \mathrm{kV}$ ). For sample preparation, aqueous dispersions of nanoparticles were brought in contact with carbon-coated $\mathrm{Cu}$ grids for $60 \mathrm{~s}$, followed by exposure to 1 $\mathrm{wt} \%$ uranyl acetate solution for $30 \mathrm{~s}$ and then dried off with a filter paper. FT-Raman spectra of nanoparticles were recorded using an FT-Raman NXR 9600 (Thermo Scientific). Before recording Raman spectra, each sample was freeze-dried using a Heto Power Dry LL 3000 (Thermo Electron Corporation). ${ }^{1} \mathrm{H}$ NMR spectra of nanoparticles were recorded using Bruker Nanobay $400 \mathrm{MHz}$. Before recording ${ }^{1} \mathrm{H}$ NMR spectra each sample was freeze-dried and re-dispersed in DMSO-d6. The determination of thiol-groups content on the surface of the nanoparticles was performed using Ellman's assay as described in our previous publications. ${ }^{12,13}$

\section{Fluorescent labeling}

For fluorescent labeling, aqueous dispersions of nanoparticles with excess of thiol groups on their surface were mixed with fluorescein-5-maleimide $(50 \mu \mathrm{mol}$ of thiol groups per $1 \mu \mathrm{mol}$ fluorescent label). The mixture was stirred continuously for 48 $\mathrm{h}$ at room temperature. Then the nanoparticles were purified by dialysis against $5 \mathrm{~L}$ deionized water (12 changes of water in total). The fluorescently labeled nanoparticles were stored in sealed containers covered with aluminum foil at $4{ }^{\circ} \mathrm{C}$. Fluorescent spectra of nanoparticles were recorded using a FP-6200 spectrofluorimeter (Jasco, UK) with $\lambda_{\mathrm{ex}}=492 \mathrm{~nm}$.

\section{Retention on mucosal surfaces}

Nanoparticles retention studies used a fluorescence microscope (Zeiss, Imager.A1) with a AxioCam MRm Zeiss camera at $5 \times$ magnification. Porcine urinary bladders were obtained from the local abattoir and were stored at $-20{ }^{\circ} \mathrm{C}$. Prior to experiments, tissue $(2 \times 3 \mathrm{~cm})$ was thawed using warm water. Background microscopy images were recorded for each mucosal tissue prior to application of $200 \mu \mathrm{L}$ nanoparticle dispersion. Microscopy images were recorded before $10 \mathrm{~mL}$ artificial urine $(2.427 \mathrm{~g}$ urea, $0.634 \mathrm{~g} \mathrm{NaCl}, 0.450 \mathrm{~g} \mathrm{KCl}, 0.161 \mathrm{~g}$ $\mathrm{NH}_{4} \mathrm{Cl}, 0.089 \mathrm{~g} \mathrm{CaCl}, 0.100 \mathrm{~g} \mathrm{MgSO}_{4}, 0.034 \mathrm{~g} \mathrm{NaHCO} .0 .0258$ $\mathrm{g}$ of $\mathrm{Na}_{2} \mathrm{SO}_{4}, 0.100 \mathrm{~g}$ of $\mathrm{NaH}_{2} \mathrm{PO}_{4}$, and $0.011 \mathrm{~g}$ of $\mathrm{Na}_{2} \mathrm{HPO}_{4}$ dissolved in $200 \mathrm{~mL}$ of deionized water to give solution with $\mathrm{pH}$ 6.2) ${ }^{14}$ was dripped onto the mucosal surface at $5 \mathrm{~mL} \mathrm{~min}^{-1}$ using a syringe pump. Microscopy images were recorded after each wash. The wash cycles were repeated 4 times and the fluorescence results are presented as a function of total volume of artificial urine used. The histograms of fluorescence distribution were obtained by analyzing the images using ImageJ in 8-bit grayscale. The mean fluorescence values (normalized intensity, a.u.) were calculated for each image from the histograms. These values were normalized by subtracting the fluorescence recorded for the biological tissues prior to deposition of nanoparticles. Each value was also normalized by dividing it by the fluorescence of deposited nanoparticles prior to their wash off with artificial urine.

\section{Results and discussion}

Solution mixtures of PEMP and PETA in DMF were prepared at different concentrations and reagent ratios and thiol-ene click reaction was allowed to proceed for $24 \mathrm{~h}$ under continuous stirring at room temperature. Depending on the total concentration as well as PEMP-PETA ratio in solutions, the click reaction resulted either in the formation of macroscopic transparent gels or solutions (sols). The gelation of reaction mixtures was confirmed by inability of magnetic bars to stir and by vial inversion. The formation of gels was typically observed for mixtures where the total concentration of PEMPPETA was above 10 vol\%. Non-stoichiometric PEMP-PETA 


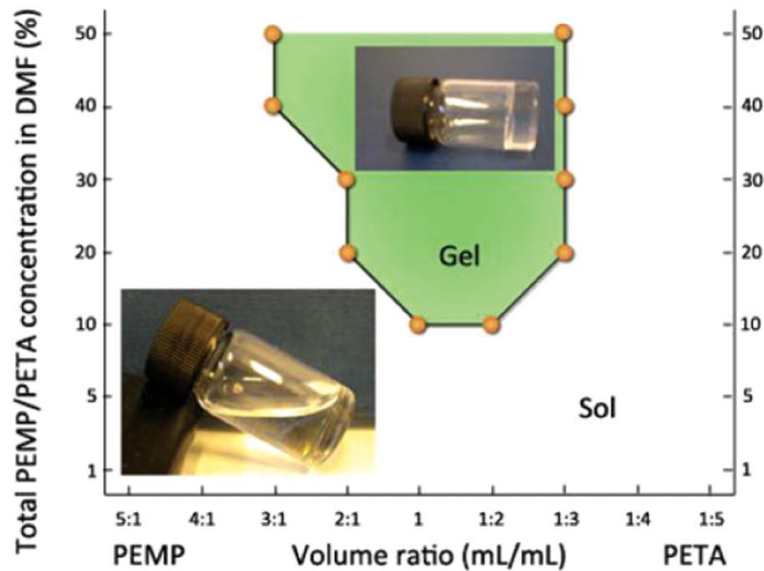

Fig. 1 Phase diagram showing the formation of cross-linked network (gel) and nanoparticles (sol) depending on PEMP-PETA ratio and concentrations in the reaction mixture. Insets: photographs of gel and sol sample immediately in the end of click reaction (before water was added to these samples).

ratios formed soft and rubbery gels, which showed some swelling in water. The gel samples exposed to excess of water gradually lost their transparency and turned white. The samples with 1:1 thiol-to-ene ratios were generally harder and stiffer compared to the gels prepared from non-stoichiometric PEMP-PETA ratios. This observation is in agreement with the report by Carlborg et al. ${ }^{3}$ Upon addition of water to the reaction mixtures resulting in transparent solutions after $24 \mathrm{~h}$ of stirring, an immediate phase separation was observed leading to the formation of milky suspensions. The formation of sols tended to result for the mixtures with less-stoichiometric ratios and with lower total concentrations of the reagents (Fig. 1 and Table 1S, ESI †). The milky suspensions resulting from addition of water to liquid reaction mixtures (sols) were purified by dialysis.

This led to the isolation of the nanoparticles, which were subsequently gravimetrically analyzed to determine their yield and dynamic light scattering/zeta-potential measurements estimated their dimensions and surface properties. The results of this analysis are summarized in Table 1 . The yields of nanoparticles were relatively low (3-16\%); higher yields were not possible because of potential tendency of PEMP-PETA more concentrated mixtures to cross-link and form gels. The

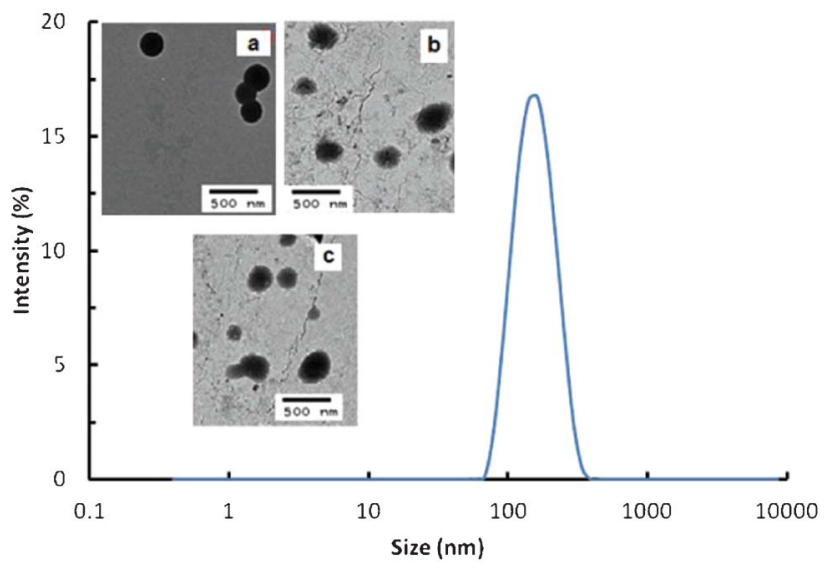

Fig. 2 DLS size distribution for PEMP-PETA (1: 1) nanoparticles. Inserts: TEM images of $1: 1$ (a), $1: 2$ (b) and $5: 1$ (c) PEMP-PETA nanoparticles.

nanoparticles were found to have relatively large sizes, between 250 and $555 \mathrm{~nm}$, low polydispersities (PDI $=0.030-$ 0.314 ) and negative zeta potentials $(\xi=-34.1$ to $-57.9 \mathrm{mV})$. These negatively charged surfaces provided nanoparticles with excellent stability towards aggregation. The thiol group content on the surface of nanoparticles, determined using Ellman's assay, ranged from 0 to $106 \pm 2 \mu \mathrm{mol} \mathrm{g}{ }^{-1}$. This was consistent with the PEMP-PETA ratio in the reaction mixtures: PETA enriched systems showed low or no presence of thiols, whereas PEMP-rich mixtures resulted in high concentrations of $-\mathrm{SH}$ groups.

Through the use of dynamic light scattering and transmission electron microscopy it was established that the nanoparticles have a monomodal size distribution (Fig. 2). They generally have spherical shape and non-porous morphology, however, the samples prepared with non-stoichiometric PEMP-PETA ratios showed poorer sphericity compared to the stoichiometric ones.

The nature of functional groups and chemical bonds in the nanoparticles were studied using Raman and ${ }^{1} \mathrm{H}$ NMR spectroscopy. The Raman spectra recorded for the samples with stoichiometric PEMP-PETA (1:1) ratios revealed strong bands at $2930 \mathrm{~cm}^{-1}$ due to $\mathrm{C}-\mathrm{H}$ stretching $\left(v_{\mathrm{CH}}\right), 1736 \mathrm{~cm}^{-1}$ due to $v_{\mathrm{C}=\mathrm{O}}, 1422 \mathrm{~cm}^{-1}$ and $1217 \mathrm{~cm}^{-1}$ due to $\delta_{\mathrm{CH} 2}, 1049 \mathrm{~cm}^{-1}$ due to $v_{\mathrm{C}-\mathrm{C}}, 936 \mathrm{~cm}^{-1}$ due to $v_{\mathrm{C}-\mathrm{O}-\mathrm{C}}, 669 \mathrm{~cm}^{-1}$ due to $v_{\mathrm{C}-\mathrm{S}}$ and

Table 1 Reaction mixtures and characteristics of the resulting nanoparticles

\begin{tabular}{|c|c|c|c|c|c|c|}
\hline Volume of DMF [mL] & PEMP-PETA volume ratio $\left[\mathrm{mL} \mathrm{mL}^{-1}\right]$ & Yield $[\%]$ & $\operatorname{Diameter}^{a}[\mathrm{~nm}]$ & $\mathrm{PDI}^{a}$ & $-\mathrm{SH}$ content $\left[\mu \mathrm{mol} \mathrm{g}^{-1}\right]$ & $\zeta[\mathrm{mV}]$ \\
\hline 25 & $3: 1$ & 13 & $428 \pm 2$ & 0.164 & $38 \pm 1$ & $-58 \pm 3$ \\
\hline 20 & $2: 4$ & 16 & $462 \pm 9$ & 0.119 & $21 \pm 2$ & $-34 \pm 1$ \\
\hline 20 & $3: 1$ & 12 & $554 \pm 5$ & 0.314 & $66 \pm 2$ & $-47 \pm 1$ \\
\hline 20 & $1: 4$ & 6 & $399 \pm 4$ & 0.260 & 0 & $-53 \pm 1$ \\
\hline 50 & $5: 1$ & 10 & $251 \pm 2$ & 0.148 & $106 \pm 2$ & $-49 \pm 3$ \\
\hline 50 & $2: 2$ & 6 & $298 \pm 4$ & 0.030 & $13 \pm 1$ & $-51 \pm 1$ \\
\hline 50 & $1: 3$ & 3 & $495 \pm 5$ & 0.030 & $4 \pm 1$ & $-39 \pm 1$ \\
\hline
\end{tabular}

${ }^{a}$ Determined using DLS. 

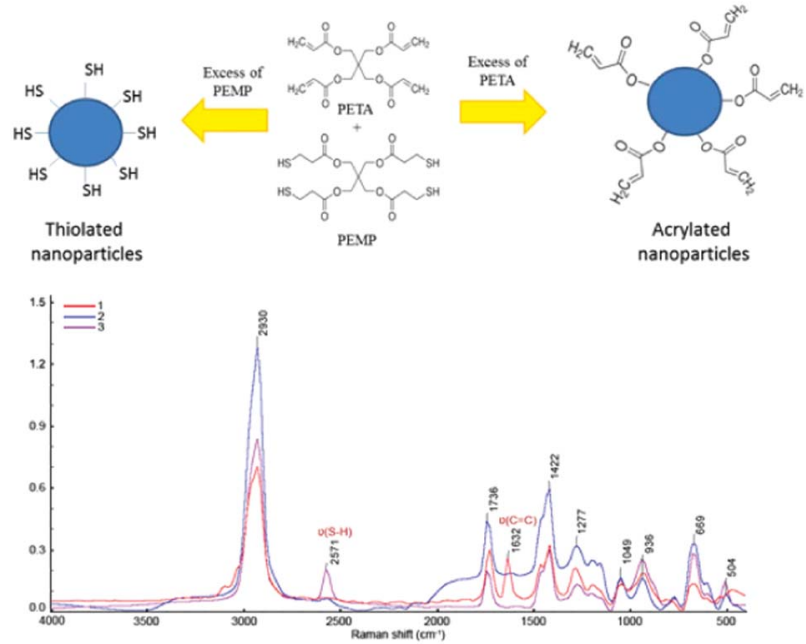

Fig. 3 Schematic structures and Raman spectra of nanoparticles with excess of PETA (1), with PETA-PEMP $1: 1$ ratio (2) and with excess of PEMP (3).

$504 \mathrm{~cm}^{-1}$ due to $v_{\mathrm{S}-\mathrm{S}}$. Raman spectra of the nanoparticles prepared with excess PEMP showed strong bands at $2571 \mathrm{~cm}^{-1}$ due to S-H stretching $\left(v_{\mathrm{SH}}\right)$. Raman spectra of the nanoparticles prepared with excess of PETA did not show any bands typical for $\mathrm{SH}$ groups, but the strong band responsible for $\mathrm{C}=\mathrm{C}$ stretching was found at $1632 \mathrm{~cm}^{-1}$. This interpretation of Raman spectra is in agreement with the literature. ${ }^{12,15}$ Fig. 3 shows Raman spectra and proposed schematic structure of both thiolated and acrylated nanoparticles.

${ }^{1} \mathrm{H}$ NMR spectroscopy was also used to further confirm that an excess of one component in the reaction mixture leads to the presence of reactive groups on the surface of nanoparticles (Fig. 1S, ESI $\dagger$ ). As predicted, ${ }^{1} \mathrm{H}$ NMR spectra of nanoparticles with excess PEMP showed a peak at $1.26 \mathrm{ppm}$ due to the protons in - $\mathrm{SH}$ groups, ${ }^{16}$ while the spectra of nanoparticles with excess PETA revealed all three alkenyl protons at 5.98 ppm, $6.18 \mathrm{ppm}$ and $6.35 \mathrm{ppm}$. Other peaks were found at 2.60$3.00 \mathrm{ppm}$ due to $-\mathrm{CH}_{2}-\mathrm{CH}_{2}-\mathrm{S}-$ groups, and at $4.12 \mathrm{ppm}$ due to $\equiv \mathrm{C}-\mathrm{CH}_{2}-\mathrm{O}$-groups.

The thiolation levels registered for PEMP-enriched nanoparticles were comparable with the $-\mathrm{SH}$ concentration found in thiolated silica reported in our previous study, ${ }^{10}$ which makes them potentially useful as mucoadhesive materials and for post-functionalization via fluorescent labelling or PEGylation.

The PEMP-PETA (5:1) nanoparticles were labelled through reaction with fluorescein-5-maleimide (see Fig. 2S, ESI $\dagger$ with the fluorescence spectra of fluorescently labelled nanoparticles). The unlabelled particles did not fluoresce.

Materials capable of adhering to mucosal tissues in the human body are currently of significant interest for the development of drug delivery systems for transmucosal administration..$^{9,17-19}$ A number of routes of drug administration can benefit from the use of mucoadhesive materials because they help to retain dosage forms in contact with biological tissues, which results in enhanced drug bioavail- ability and improved efficiency of therapy. The typical transmucosal routes of drug administration include nasal, ocular, oromucosal, vaginal and rectal.

Intravesical route of drug administration is currently used for the delivery of chemotherapeutical agents directly into urinary bladder to treat cancer. ${ }^{20,21}$ This route can also benefit from drug nano-carriers capable of adhering to urinary bladder mucosal surfaces. The ability of nano-carriers to adhere to mucosal epithelia will potentially improve drug retention in the urinary bladder because it will withstand drug wash-out effects related to urine voiding. Recently, thiolated nanoparticles prepared by ionic gelation of thiolated chitosan with tripolyphosphate were reported by Bernkop-Schnürch et al. to be useful for intravesical delivery. ${ }^{22}$

The ability of our thiolated nanoparticles to adhere to mucosal surfaces was tested in vitro using porcine urinary bladder tissues. To test retention, the dispersion of fluorescently-labeled PEMP-PETA (5:1) was placed on mucosal surface and was washed off with different volumes of artificial urine. The retention of the nanoparticles on mucosal surface was evaluated using fluorescent microscopy with subsequent image analysis. Fig. 4 shows the fluorescent microphotographs of porcine urinary bladder tissues with fluorescent nanoparticles and fluorescently-labeled dextran (FITC-dextran), and also retention profiles obtained using analysis of these images. FITC-dextran was used as a negative control because of its poor mucoadhesive characteristics. ${ }^{9}$

The co-existence of both green (fluorescent) and dark (nonfluorescent) regions in some of the images may be related to different affinity of microscopic regions of a biological tissue to the nanoparticles (potentially uneven distribution of thiols on mucosal tissue). The thiolated nanoparticles exhibited
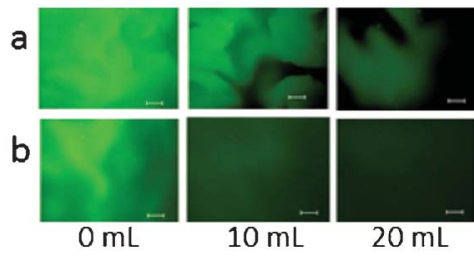

$20 \mathrm{~mL}$

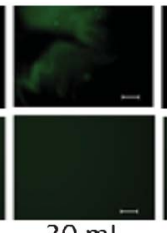

$30 \mathrm{~mL}$
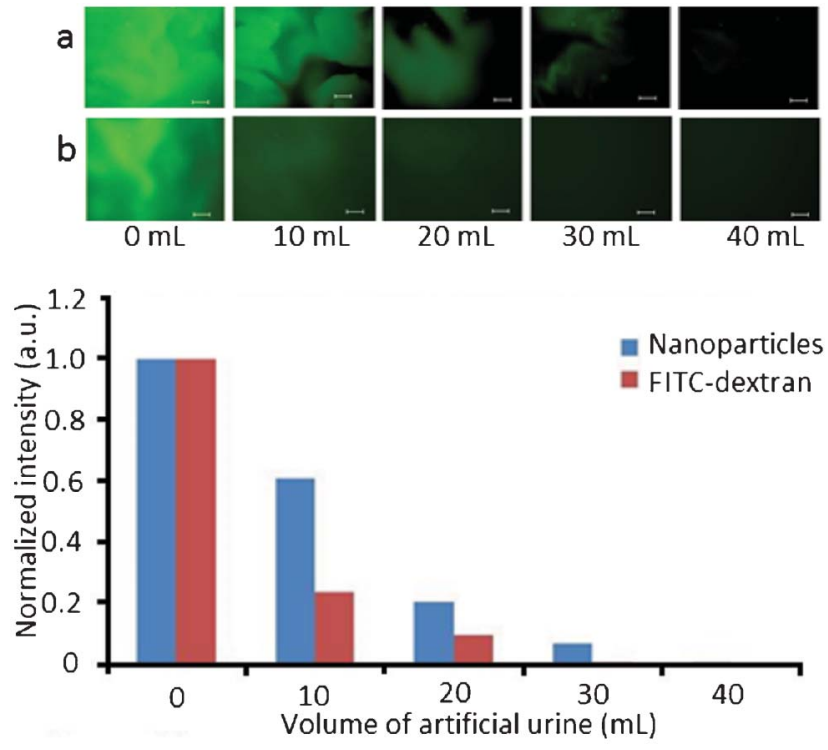

Fig. 4 Fluorescence levels of thiolated nanoparticles and FITC-dextran on porcine urinary bladder surfaces washed with artificial urine. Inserts show fluorescent microphotographs of porcine urinary bladder surfaces with thiolated nanoparticles (a) and FITC-dextran (b). Size bar is $200 \mu \mathrm{m}$. 
significantly better retention on mucosal surfaces compared to FITC-dextran, which confirms their mucoadhesive properties.

The presence of numerous ester linkages in these nanoparticles also implies that they may undergo hydrolytic degradation. To test this, an aqueous dispersion of nanoparticles (PEMP-PETA ratio of $1: 1$ ) was stored at room temperature for 90 days and analyzed using dynamic light scattering. A substantial reduction in the particle size from 170 to $146 \mathrm{~nm}$ is observed over this time, accompanied by dramatic change in the particle size distribution (Fig. 3S, ESI $\dagger$ ). The slow hydrolytic degradation of these nanoparticles could be of interest for developing delivery systems with sustained drug release profiles.

The advantage of these thiol-ene click nanoparticles over silica-based nanomaterials reported in our previous publications ${ }^{12,13}$ is their swellability in organic solvents. This swellability may potentially be used for loading drugs into these nanoparticles.

\section{Conclusions}

Thiol-ene click reactions between pentaerythritol tetrakis(3mercaptopropionate) and pentaerythritol tetraacrylate in $\mathrm{N}, \mathrm{N}$-dimethylformamide were found to produce nanoparticles, whose surface functionality is greatly dependent on the reagents ratio in the reaction mixtures. The possibility of preparing either thiolated or acrylated nanoparticles was demonstrated when non-stoichiometric reagent ratios were used. These nanoparticles undergo gradual hydrolytic degradation, which may find applications for drug delivery, e.g. for designing novel nanomedicines to provide sustained drug release. The nanoparticles with surface functional groups could be further functionalized. The ability to label thiolated nanoparticles fluorescently was demonstrated through reaction with fluorescein maleimide. Acrylated nanoparticles could also be potentially used for post-functionalization, e.g. through the reaction with thiol-bearing proteins.

The thiolated nanoparticles were mucoadhesive similarly to thiol-bearing polymers that are widely used in the development of novel dosage forms for transmucosal drug delivery. ${ }^{7,8}$ Therefore the thiol-ene click approach to synthesize thiolated nanoparticles could be considered as a novel alternative for preparing mucoadhesive materials through chemical conjugation of SH-bearing moieties to water-soluble polymers. The acrylated nanoparticles could also be potentially mucoadhesive as they should be capable of interacting with thiol groups present in mucins, similarly to acrylated polymers recently reported by Davidovich-Pinhas and Bianco-Peled. ${ }^{23}$

\section{Acknowledgements}

AS is grateful to the ERASMUS scheme for funding her 4-months placement at the University of Reading. The authors are grateful to Chemical Analysis Facility (University of Reading) for providing access to Raman and ${ }^{1} \mathrm{H}$ NMR spectroscopy. Prof N. Tirelli and Dr F. Cellesi (University of Manchester, UK) are gratefully acknowledged for access to zeta-potential measurements. Centre for Advanced Microscopy (CfAM, University of Reading) and Dr Peter Harris are acknowledged for access and help with transmission electron microscopy. The authors are grateful to Prof Adrian Williams for providing useful comments on this manuscript.

\section{References}

1 C. E. Hoyle and C. N. Bowman, Angew. Chem., Int. Ed., 2010, 49, 1540.

2 A. B. Lowe, Polym. Chem., 2010, 1, 17.

3 C. F. Carlborg, T. Haraldsson, K. Oberg, M. Malkoch and W. van der Wijngaart, Lab Chip, 2011, 11, 3136.

4 S. Aryal, C.-M. J. Hu and L. Zhang, Small, 2010, 6, 1442.

5 G. Delaittre, G. Justribó-Hernández, R. J. M. Nolte and J. J. L. M. Cornelissen, Macromol. Rapid Commun., 2011, 32, 19.

6 J. V. Jokerst, T. Lobovkina, R. N. Zare and S. S. Gambhir, Nanomedicine, 2011, 6, 715.

7 A. Bernkop-Schnurch, Adv. Drug Delivery Rev., 2005, 57, 1569.

8 F. Laffleur and A. Bernkop-Schnurch, Future Med. Chem., 2012, 4, 2205.

9 V. V. Khutoryanskiy, Macromol. Biosci., 2011, 11, 748.

10 G. Shahnaz, A. Vetter, J. Barthelmes, D. Rahmat, F. Laffleur, J. Iqbal, G. Perera, W. Schlocker, S. Dünnhaput, P. Augustijns and A. Bernkop-Schnürch, Int. J. Pharm., 2012, 428, 164.

11 B. Deutel, M. Greindl, M. Thaurer and A. BernkopSchnürch, Biomacromolecules, 2008, 9, 278.

12 G. S. Irmukhametova, G. A. Mun and V. V. Khutoryanskiy, Langmuir, 2011, 27, 9551.

13 G. S. Irmukhametova, B. J. Fraser, J. L. Keddie, G. A. Mun and V. V. Khutoryanskiy, Langmuir, 2012, 28, 299.

14 S. Chutipongtanate and V. Thongboonkerd, Anal. Biochem., 2010, 402, 110.

15 J. H. Hibben, The Raman effect and its chemical applications, Reinhold Publishing corporation, New York, USA, 1939, pp. 125-262.

16 S. A. Richards and J. C. Hollerton, Essential Practical NMR for Organic Chemistry, John Wiley \& Sons, 2011, p. 1.

17 J. Smart, Adv. Drug Delivery Rev., 2005, 57, 1556.

18 L. Serra, J. Domenech and N. A. Peppas, Eur. J. Pharm. Biopharm., 2009, 71, 519.

19 G. Andrews, T. P. Laverty and D. S. Jones, Eur. J. Pharm. Biopharm., 2009, 71, 505.

20 S. G. Sarkar and R. Banerjee, J. Controlled Release, 2010, 148, 147.

21 C. C. Hsu, Y. C. Chuang and M. B. Chancellor, Int. J. Urol., 2013, 20, 552.

22 J. Barthelmes, G. Perera, J. Hombach, S. Dünnhaupt and A. Bernkop-Schnürch, Int. J. Pharm., 2011, 416, 339.

23 M. Davidovich-Pinhas and H. Bianco-Peled, Acta Biomater., 2011, 7, 625. 